\title{
Standing assistance system for rehabilitation walker
}

\author{
Daisuke Chugo $^{1}$ Wataru Matsuoka ${ }^{1}$ Songmin Jia ${ }^{1}$ Kunikatsu Takase ${ }^{1}$ and \\ Hajime Asama² \\ 1 The University of Electro-Communications, 1-5-1, Chofugaoka, Chofu, Tokyo, \\ Japan \{chugo, matsuoka, jia, takase\}@is.uec.ac.jp \\ 2 The University of Tokyo, 5-1-5, Kashiwanoha, Kashiwa-shi, Chiba, Japan \\ asama@race.u-tokyo.ac.jp
}

Summary. This paper proposes a walker system with power assistance device for standing up motion. Our system focuses on domestic uses for aged person who needs nursing in their daily life. Our key ideas are two topics. The first topic is new assistance mechanism with four parallel linkages. Our proposed manipulator mechanism requires only smaller actuators and realizes rigid structure with lighter linkages comparing with general manipulator. Thus, we can design our assistance system compactly with low-cost using our mechanism. The second topic is the combination of force and position control. According to the patient's posture during standing up, our control system selects more appropriate control method from them. We use the reference of standing-up motion which is based on the typical standing up motion by nursing specialist for realizing the natural assistance. The performance of our proposed assistance system is verified by computer simulations and experiments using our prototype.

Key words: Walker; Standing up motion; Force control; Position control,

\section{Introduction}

In Japan, the population ratio of senior citizen who is 65 years old or more exceeds 20[\%] at January 2004 and rapid aging in Japanese society will advance in the future. [1] In aging society, many elderly people cannot perform normal daily household, work related and recreational activities because of decrease in force generating capacity of their body. Today, the 23.5[\%] of elderly person who does not stay at the hospital cannot perform daily life without nursing by other people. [2] For their independent life, they need assistance system which enable them to perform daily life easily in their home even if their physical strength reduces.

Standing up motion is the most serious and important operation in daily life for elderly person who doesn't have enough physical strength. [3, 4] In 
typical bad case, elderly person who doesn't have enough physical strength will cannot operate standing up motion and will falls into the wheelchair life or bedridden life. Furthermore, if once elderly person falls into such life, the decrease of physical strength will be promoted because he will not use his own physical strength. [5] Therefore, force assistance system is required to use part of the remaining strength of the patient for standing-up motion in order not to reduce their muscular strength.

In previous works, many researchers developed power assistance devices for standing up motion. However, these devices are large scale and they are not suitable for family use. [6, 7] Furthermore, these devices assist all necessary power for standing up and they do not discuss the using the remaining physical strength of patients. Therefore, there is a risk of promoting the decrease of their physical strength.

In this paper, we develop a walker system with power assistance device for standing up motion. Our system is based on a walker which is popular device for aged person in their daily life and realizes the standing up motion using the support pad which is actuated by our developed assistance mechanism with three degrees of freedom. For using the remaining physical strength, our system uses the motion pattern which is based on the typical standing up motion by nursing specialist as control reference. [9]

Our key ideas are two topics. The first is new assistance mechanism with four parallel linkages which enables the system to be rigid and compact. The second is the combination of force and position control. Using our control scheme, the patients can stand up with fewer loads and can use their own remaining physical strength during the motion. We verify the performance of our proposed assistance system through simulations and experiments using our prototype.

\section{System Configuration}

\subsection{Assistance Mechanism}

Fig.1 shows our proposed assistance system. The system consists of support pad with three degrees of freedom and the walker system. (Fig.1(a)) The support pad is actuated by our new assistance mechanism with four parallel linkages.

Fig.1(b) shows the frame-kinematic model of our assistance mechanism. The position of the support pad (2DOF, red lines in Fig.1(a)) is coordinated by Actuator 1 and Actuator 2 which are equipped on $\mathrm{O}$ point. Actuator 1 drives Link1 $(\alpha)$ and Actuator 2 drives Link $2(\beta)$. Using four parallel linkages mechanism, two actuators can generate the position of support pad. The inclination of the support pad (1DOF, the blue line in Fig.1(a)) is coordinated by Actuator 3 which is equipped on $\mathrm{P}$ point. 
The advantages of our proposed mechanism are two topics. The first is that two main actuators (Actuator 1 and 2) keep only weight of linkages. In general manipulator, the actuator of lower part supports not only weight of linkages but also actuators of upper part. Therefore, the actuators of lower part are required high output traction and tend to be heavy. On the other hand, using our mechanism, main actuators are mounted on the walker body (O point) and they are required to keep only weight of linkages. As the result, we can use smaller actuators for our developed assistance mechanism.

The second is parallel linkage mechanism. The parallel linkage mechanism is rigid for a twist load, therefore, system realizes same strength with lighter linkages comparing with the general manipulator. Using our proposed mechanism, we can use smaller actuators and lighter linkages, and our system realizes compact design with low cost.

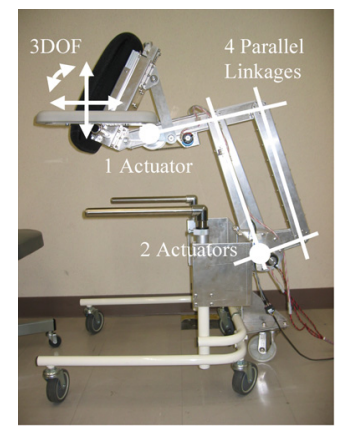

(a) Prototype system

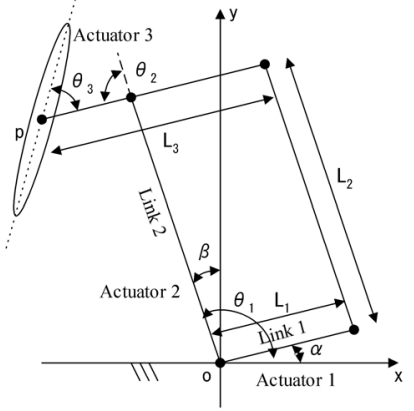

(b) Parallel Linkage Mechanism

Fig. 1. Our assistance system

\subsection{Kinematics}

In this section, we derive the inverse kinematics of our proposed linkage mechanism. In our proposed mechanism, we can derive the position of $\mathrm{P}$ point (We define its coordinate as $\left.\left(x_{p}, y_{p}\right)\right)$ using $\theta_{1}$ and $\theta_{2}$. However, it is $\alpha$ and $\beta$ that is used to control the actuators. Therefore, we set angular values and length of linkages as in Fig.1(b).

$$
\begin{array}{cc}
\theta_{1}=\frac{\pi}{2}+\beta, & \theta_{2}=\frac{\pi}{2}+\alpha-\beta \\
l_{1}=L_{2}, & l_{2}=L_{3}-L_{1}
\end{array}
$$

Now, we consider the geometric relationships among the position of $\mathrm{P}$ point and these angular values, we can derive (3) and (4). From (3) and (4), $\theta_{2}$ is (5) 


$$
\begin{gathered}
x_{p}=l_{1} \cos \theta_{1}+l_{2} \cos \left(\theta_{1}+\theta_{2}\right) \\
y_{p}=l_{1} \sin \theta_{1}+l_{2} \sin \left(\theta_{1}+\theta_{2}\right) \\
\theta_{2}=\arccos \left(\frac{x_{p}^{2}+y_{p}^{2}-l_{1}^{2}-l_{2}^{2}}{2 l_{1} l_{2}}\right)
\end{gathered}
$$

We set $k_{1}$ and $k_{2}$ as (6), (3) and (4) are expressed as (7) and (8).

$$
\begin{gathered}
k_{1}=l_{1}+l_{2} \cos \theta_{2}, \quad k_{2}=l_{2} \sin \theta_{2} \\
x_{p}=k_{1} \cos \theta_{1}-k_{2} \sin \theta_{1} \\
y_{p}=k_{1} \sin \theta_{1}+k_{2} \cos \theta_{1}
\end{gathered}
$$

Furthermore, we set $r$ and $\gamma$ as (9), $k_{1}$ and $k_{2}$ are expressed as (10). Using (10), (7) and (8) are expressed as (11) and (12).

$$
\begin{gathered}
r=\sqrt{x_{p}^{2}+y_{p}^{2}}, \quad \tan \gamma=\frac{k_{2}}{k_{1}} \\
k_{1}=r \cos \gamma, \quad k_{2}=r \sin \gamma \\
x_{p}=r \cos \left(\gamma+\theta_{1}\right) \\
y_{p}=r \sin \left(\gamma+\theta_{1}\right)
\end{gathered}
$$

From (11) and (12), we can derive (13).

$$
\tan \left(\gamma+\theta_{1}\right)=\frac{y_{p}}{x_{p}}
$$

Thus, $\theta_{1}$ is (14). Using (5) and (14), we can control our mechanism.

$$
\theta_{1}=\arctan \left(\frac{y_{p}}{x_{p}}\right)-\arctan \left(\frac{l_{2} \sin \theta_{2}}{l_{1}+l_{2} \cos \theta_{2}}\right)
$$

\section{Motion Control}

\subsection{Motion by Nursing Specialist}

In previous study, a lot of standing up motions for assistance are proposed. Kamiya [10] proposed the standing up motion which uses remaining physical strength of the patients maximum based on her experience as nursing specialist. In our previous work, we analyze this standing up motion and find that Kamiya scheme is effective to enable standing up motion with smaller load. [9]

Thus, we measure the angular values among the linkages, which reflect the relationship of body segments during standing up motion with Kamiya 
scheme. The angular value is derived using the body landmark as shown in Fig.2(a). We assume the standing up motion is symmetrical and we discuss the motion as movement of the linkages model on 2D plane. [8]

From measuring results, the trunk needs to incline to forward direction during lifting up from chair as shown in Fig.2(b). Y-axis shows the angular value (Pelvis and trunk, knee, ankle) and $\mathrm{X}$-axis shows the movement pattern $(\hat{s})[14]$ which means the ratio of standing up operation as (15).

$$
\hat{s}=\frac{t}{t_{s}}
$$

where $t_{s}$ is required time to the standing up operation and $t$ is present time.

In order to realize the natural standing up motion by Kamiya scheme, we use these results as track references.

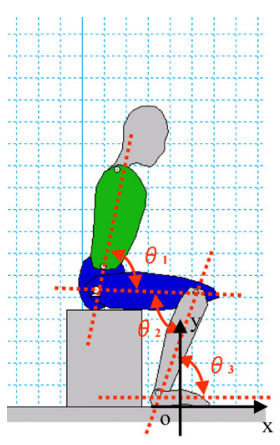

(a) Coordination

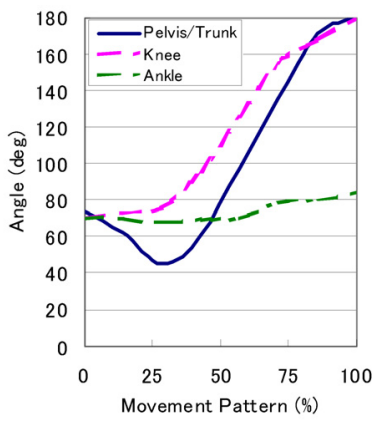

(b) Angular Value

$\theta_{1}, \theta_{2}$ and $\theta_{3}$ show the angles of pelvis/trunk, knee and ankle, respectively.

Fig. 2. Standing-up motion with Kamiya scheme

\subsection{Derivation of Control Reference}

In this section, we derive the control reference of our assistance system which can realize the standing up motion proposed by Kamiya using a computer simulation. Fig.3(a) shows the simulation setup. The parameters are chosen from a standard body data of adult male as shown in Table 1. [11][12] We use the Working Model 2D as a physical simulator and MATLAB as a controller. Both applications are linked by Dynamic Data Exchange function on Windows OS.

From these simulation results, Fig.3(b) shows position references of the support pad and Fig.3(c) shows reference angle. The coordination is defined as shown in Fig.3(a). In Fig.3(b), the start point is lower and the end point is upper. Using these tracks as the position control reference, our assistance system can realize the standing up motion which Kamiya proposes. 


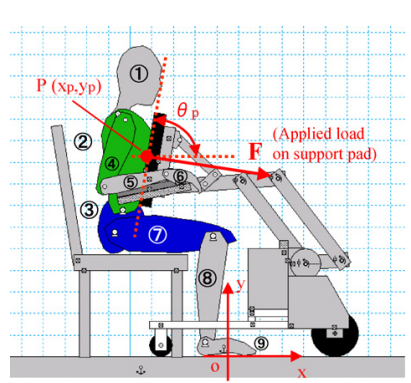

(a) Simulation Setup

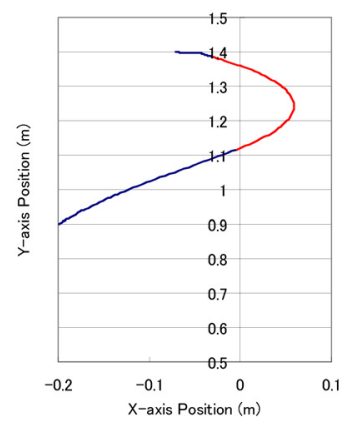

(b) Position Reference

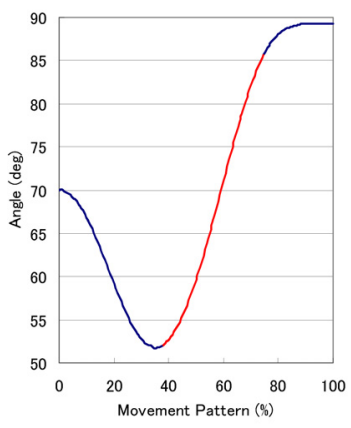

(c) Angle Reference Red line shows the force control mode. (We discuss in next section.)

Fig. 3. Derived control references

Table 1. Human Parameters

\begin{tabular}{|c|c|c|c|c|}
\hline Number & Link Name & Mass $[\mathrm{kg}]$ & Length $[\mathrm{m}]$ & Width $[\mathrm{m}]$ \\
\hline 1 & Head & 5.9 & 0.28 & 0.21 \\
\hline 2 & Trunk & 27.2 & 0.48 & 0.23 \\
\hline 3 & Hip & 18.1 & 0.23 & 0.23 \\
\hline 4 & Humerus & 4.5 & 0.39 & 0.12 \\
\hline 5 & Arm & 2.7 & 0.35 & 0.08 \\
\hline 6 & Hand & 0.5 & 0.2 & 0.07 \\
\hline 7 & Femur & 9.1 & 0.61 & 0.17 \\
\hline 8 & Leg & 4.5 & 0.56 & 0.16 \\
\hline 9 & Foot & 0.8 & 0.26 & 0.11 \\
\hline
\end{tabular}

\subsection{Force Control}

For realizing the motion of Kamiya scheme using the remaining physical strength of patients, the required condition for standing-up motion is different at each phase during standing up motion. [9]

- When the patient lifts up his body, it is required to reduce the load of knee joint. Therefore, it is required to force assistance.

- In other phases, it is required to maintain the standing up motion. Therefore, it is required to position maintaining assistance.

In order to fulfill both conditions, we propose new control scheme. Proposed control scheme combines dumping control and position control. The dumping control is suitable for the control of the objects with contact [13]. We use a force sensor attached on the support pad ( $\mathrm{P}$ point in Fig.3(a)) for switching condition between the position control and the dumping control.

$$
v_{i}=v_{i}^{r e f}-B\left(F-F_{0}\right)-K\left(x_{i}-x_{i}^{r e f}\right)
$$


where $F$ is the applied force on the support pad as shown in Fig.3(a). $F_{0}$ is the threshold which selects force or position control. $v_{i}^{r e f}$ is the velocity reference and $x_{i}^{\text {ref }}$ is the position reference derived by reference tracks in Fig.3(b) and (c). $v_{i}$ is actucal control velocity reference. $B$ and $K$ are constants.

Using this control scheme, when the patient lifts up his body and the load is large, he will lean on the support pad aggressively and the applied force on the support pad will be large. Therefore, our system can detect suitable condition between the position control and the dumping control.

\subsection{Computer Simulation}

We verify the performance of our control scheme by the computer simulation. In this experiment, the human model stands up with Kamiya motion and our assistance system assists him using our proposed control scheme. Furthermore, we compare the result by our proposed scheme with the result using only the position control reference.

We use the control references as shown in Fig.3(b) and (c) which are derived from standing up motion with Kamiya scheme in previous section. The simulation parameters are chosen from Table 1 . The coordination is defined in Fig.3(a). Furthermore, we set $F_{0}=150[\mathrm{~N}]$ as a threshold which is derived experimentally. Red lines in Fig.3(b) and (c) show that the system selects the force control.

Fig.4 shows the simulation results. Fig.4 is standing up motion using our proposed assistance control. Allows in Fig. 4 show the applied assistance force to the patient. The support pad applies the force vertically to the chest and the hand. Using our proposed control scheme, we verified that our assistance system realizes the natural standing up motion.

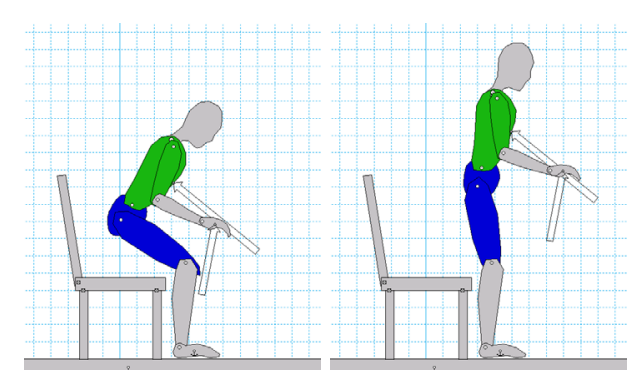

Fig. 4. Simulation Results

Table 2 shows the maximum output and output power of each joint. From these results, our proposed control scheme reduces maximum output into $0.5[\mathrm{Nm} / \mathrm{kg}]$ comparing with the result by the position control. In general, if the applied load to each joint is heavier than $0.5[\mathrm{Nm} / \mathrm{kg}]$, it is difficult to 
stand up for the elderly person. [15] Therefore, the patient can adapt this motion using his remaining physical strength.

Furthermore, with our proposed control scheme, the workload of each joint is maintained comparing with the result by the position control. During standing up motion, the patient is required to use his 92[\%] of physical strength comparing with physical strength without the force assistance control. This means our assistance system can use part of his remaining strength in order not to reduce muscular strength. Therefore, we can verify that the system selects more appropriate control method and our proposed control scheme is effective.

Table 2. Simulation Results

\begin{tabular}{|c|c|c|c|c|}
\hline \multicolumn{2}{|c|}{} & Pelvis/Trunk & Knee & Ankle \\
\hline \multirow{2}{*}{ Only Position Control } & Peak(Nm/kg) & 0.48 & 0.59 & 0.38 \\
\cline { 2 - 5 } & Workload(Ws) & 29.0 & 39.3 & 31.0 \\
\hline \multirow{2}{*}{ Proposed Control } & Peak(Nm/kg) & 0.49 & 0.50 & 0.38 \\
\cline { 2 - 5 } & Workload(Ws) & 26.9 & 36.3 & 28.8 \\
\hline
\end{tabular}

\section{Experiment}

Here, we verify the performance of our prototype system by the experiment. In this experiment, our prototype system assists the patient with control references shown in Fig.3(b) and (c). As the result of the experiment, our system can assist the patient as shown in Fig.5. The height of the patient is $170[\mathrm{~cm}]$ and the system lifts him at $30[\mathrm{sec}]$.
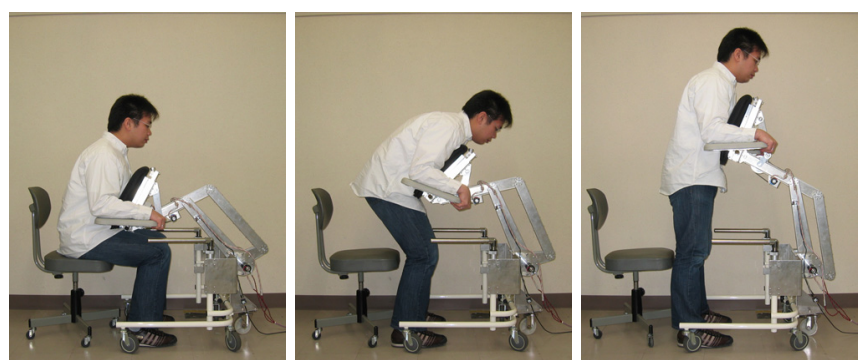

Fig. 5. Experimental results

Fig.6 shows the tracks of the position of the patient's waist, knee and ankle joint and their control references. From Fig.6, both tracks are almost same line and this means our assistance system realizes the natural standing-up motion by nursing specialist. 


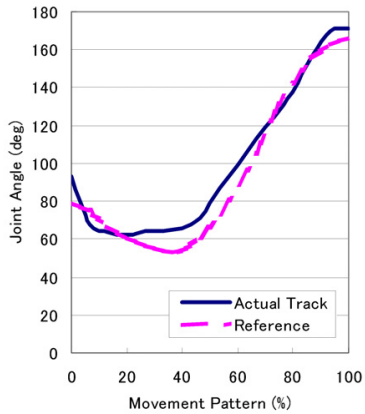

(a) Pelvis / Trunk

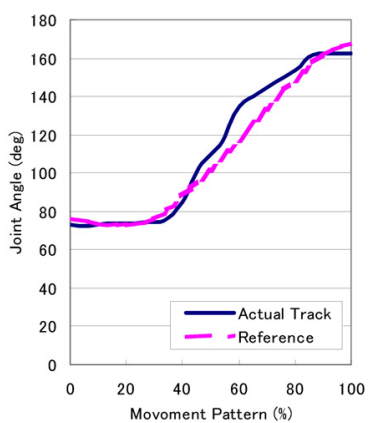

(b) Knee

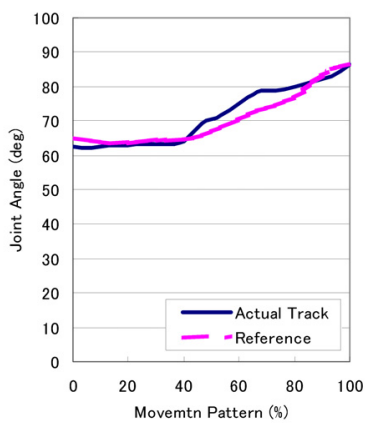

(c) Ankle

Fig. 6. Angular value of each joint

\section{Conclusion}

In this paper, we develop the novel assistance system for the standing up motion. Our system focuses on family use and system can assist the elderly person using part of their remaining strength, in order not to reduce muscular strength.

In order to fulfill this condition, we propose new assistance manipulator mechanism with parallel linkages. Our developed mechanism enables the assistance system to be rigid and compact with low costs.

Furthermore, we design the novel control scheme which combines the dumping and the position control. According to the posture of the patient during standing up motion, our control system can select more appropriate control method from them. Using our assistance system, the load of knee joint reduces to $0.5[\mathrm{Nm} / \mathrm{kg}]$, which the elderly person can adapt with their own physical strength, generally. Our system requires the patient to use his $92[\%]$ of physical strength comparing with physical strength without the force assistance control. This means our assistance system can use part of his remaining strength in order not to reduce muscular strength.

In our future work, we will develop the active walker function, which enables to assist the patients during standing, walking and seating motion continuously.

\section{Acknowledgement}

This study is supported in part by France Bed Medical Home Care Research Subsidy Foundation. 


\section{References}

1. Statistics Bureau, Ministry of Internal Affairs and Communications, Japan : " Current Population Estimates as of October 1, 2004," 2004. http://www.stat.go.jp/english/data/jinsui/2004np/index.htm (Available at February 2007)

2. Ministry of Health, Labour and Welfare, Japan: "Annual Reports on Health and Welfare 2001 Social Security and National Life," 2001. http://www.mhlw.go.jp/toukei/saikin/hw/k-tyosa/k-tyosa01/4-3.html (Available at February 2007)

3. N. B. Alexander, A. B. Schultz and D. N. Warwick, "Rising From a Chair: Effects of Age and Functional Ability on Performance Biomechanics," J. of Geometry: MEDICAL SCIENCES, Vol.46, No.3, M91-98, 1991

4. M. A. Hughes, M. L. Schenkman, "Chair rise strategy in the functionally impaired elderly," J. of Rehabilitation Research and Development, Vol.33, No.4, pp.409-412, 1996

5. M. Hirvensalo, T. Rantanen and E. Heikkinen, "Mobility difficulties and physical activity as predictors of morality and loss of independence in the communityliving older population," J. of the American Geriatric Society, Vol.48, pp.493498, 2000.

6. K. Nagai, I. Nakanishi and H. Hanabusa, "Assistance of self-transfer of patients using a power-assisting device," Proc. of the IEEE Int. Conf. on Robotics and Automation, pp.4008-4015, 2003.

7. A. Funakubo, H. Tanishiro, Y. Fukui, "Power Assist System for Transfer Aid," J. of the Society of Instrument and Control Engineers, Vol.40, No.5, pp.391-395, 2001.

8. S. Nuzik, R. Lamb, A. Vansant and S. Hirt, "Sit-to-Stand Movement Pattern, A kinematic Study," Physical Therapy, Vol.66, No.11, pp.1708-1713, 1986.

9. D. Chugo, E. Okada, K. Kawabata, H. Kaetsu, H. Asama, N. Miyake and K. Kosuge, "Force Assistance Control for Standing-Up Motion," Proc. of IEEE/RASEMBS Int. Conf. on Biomedical Robotics and Biomechatoronics, F132, 2006.

10. K. Kamiya, " Development and evaluation of life support technology in nursing," Proc. of 7th RACE Symp., Research into Intelligent Artifacts for the Generalization of Engineering, The University of Tokyo, pp.116-121, 2005.

11. Digital Human Research Center, AIST, "Human Body Properties Database," http://www.dh.aist.go.jp/bodyDB/index-e.html (Available at March 2006)

12. K. Omori, Y. Yamazaki, H. Yokoyama, U. Aoki, M. Kasahara, K. Hiraki, "The relationship between strength in the lower extremity and the ability to stand up from a chair in elderly inpatients," Sogo Rehabilitation, Vol.30, No.2, pp.167171, 2001.

13. T. Sugihara, K. Kawabata, H. Kaetsu, H. Asama, K. Kosuge and T. Mishima, "Development of a Reasonable Force Sensor for a Standing-up and Sitting Motion Support System," Proc. of Robotics and Mechatronics Conf. 2004, 1P1-H11, 2004.

14. M. Schenkman, R. A. Berger, P. O. Riley, R. W. Mann and W. A. Hodge, "Whole-Body Movements During Rising to Standing from Sitting," Physical Therapy, Vol.70, No.10, pp.638-648, 1990.

15. N. M. Fisher, D. R. Pendergast, E. C. Calkins, "Maximal Isometric Torque of Knee Extension as a Function of Muscle Length in Subjects of Advancing Age," Arch Phys Med Rehabil, Vol.71, No.10, pp.729-734, 1990. 